\title{
Effets de la fertilisation organo- minérale sur la croissance et le rendement du « mil sanio » (Pennisetum glaucum L. R. Br) en Haute Casamance (Sénégal)
}

\begin{abstract}
Abdou Ndiaye, Ousmane Ndiaye,

Université Assane Seck Ziguinchor, Département d'Agroforesterie, Sénégal Baboucar Bamba, Institut Sénégalais de Recherches Agricoles (ISRA/Dijbelor/Ziguinchor)

Moustapha Guèye, Institut Sénégalais de Recherches Agricoles (CNRA/Bambey)

Ousmane Sawané,

Université Assane Seck Ziguinchor, Département d'Agroforesterie, Sénégal

Doi:10.19044/esj.2019.v15n33p155 URL:http://dx.doi.org/10.19044/esj.2019.v15n33p155

\section{Résumé}

La présente étude vise à identifier le meilleur plan de fumure organominérale pour une bonne production en grains du mil Sanio au Sud du Sénégal. Un dispositif expérimental en split-plot avec 03 répétitions a été mis en place. Le facteur principal est la fertilisation minérale (FM) avec quatre niveaux (FM1, FM2, FM3 et FM4) et le facteur secondaire, l'amendement organique $(\mathrm{AO})$ avec trois traitements $(\mathrm{AO} 1, \mathrm{AO} 2$ et $\mathrm{AO} 3)$. Les résultats ont montré qu'à maturité les tailles des plantes les plus élevées ont été obtenues avec les apports AO3 $(291,1 \mathrm{~cm})$ et FM4 $(284,2 \mathrm{~cm})$. Le tallage le plus important a été noté avec le traitement FM4 (15 talles/poquet). Des gains respectifs de $26 \%, 32 \%$ et $56 \%$ du rendement en biomasse aérienne sèche sont obtenus avec l'augmentation des doses d'engrais minéral. Les rendements en grains les plus élevés sont obtenus avec les apports FM2 et FM4 avec des taux d'accroissements respectifs de $37,1 \%$ et $62,3 \%$ par rapport au témoin non fertilisé. Dans le cadre de l'amélioration durable de la productivité du mil sanio en Haute Casamance, l'association AO2 et FM2 d'engrais minéral serait une pratique à vulgariser.
\end{abstract}

Mots clés : Fertilisation organo-minérale, Croissance, Rendement, Mil sanio (Pennisetum glaucum (L.) R. Br) 


\title{
The Effet of Organo-Mineral Fertilization on Growth and Yield of the Sanio Millet (Pennisetum Glaucum L. R. Br) in High Casamance (Sénégal)
}

\author{
Abdou Ndiaye, \\ Ousmane Ndiaye, \\ Université Assane Seck Ziguinchor, Département d'Agroforesterie, Sénégal \\ Baboucar Bamba, \\ Institut Sénégalais de Recherches Agricoles (ISRA/Dijbelor/Ziguinchor) \\ Moustapha Gueye, \\ Institut Sénégalais de Recherches Agricoles (CNRA/Bambey) \\ Ousmane Sawane,
}

Université Assane Seck Ziguinchor, Département d'Agroforesterie, Sénégal

\begin{abstract}
This study aims to identify the best organo-mineral fertilization plan for a good grain production of sanio millet. The experiment was laid out in a split-plot design with three repetitions. The main factor is the mineral fertilization (FM) with four levels (FM1, FM2, FM3 and FM4) and a secondary factor, the organic amendment $(\mathrm{AO})$ with three treatments (AO1, $\mathrm{AO} 2$ and AO3). The results showed that at maturity the highest plant sizes were obtained with AO3 $(291.1 \mathrm{~cm})$ and FM4 $(284.2 \mathrm{~cm})$ inputs. The most important tillering was noted with the FM4 treatment (15 tillers / pouch). Increase respective of $26 \%, 32 \%$ and $56 \%$ of biomass production were obtained with the increase of the mineral fertilizer doses. The highest grain yields are obtained with the FM3 and FM4 inputs with respective growth rates of $37.1 \%$ and $62.3 \%$ compared to the control. In the context of the sustainable management of the productivity of the sanio millet in Casamance higher land, the the combination of $\mathrm{AO} 2$ and FM2 of mineral fertilizer could be advised to farmers.
\end{abstract}

Keywords: Organo-Mineral Fertilization, Growth, Yield, Sanio Millet (Pennisetum Glaucum (L.) R. Br) 


\section{Introduction}

Le mil occupe une place importante dans la production céréalière mondiale. En effet, il est la quatrième céréale la plus cultivée derrière le blé, le riz et le maïs (FAO, 2017). La production mondiale de mil était estimée à 28459020 tonnes sur une superficie de 3124442 ha avec un rendement moyen: de $910 \mathrm{~kg} / \mathrm{ha}(\mathrm{FAO}, 2017)$. Plus de $80 \%$ de la production mondiale du mil provient de l'Afrique et de l'inde (FAO, 2016). Au Sénégal, il représente la céréale la plus importante du point de vue de la production (ANSD, 2015; FAO, 2016). Sa production en 2015 était de 749874 tonnes et représentait environ $31 \%$ de la production céréalière nationale (DAPSA 2016). Dans la région de Kolda (haute Casamance) la production de mil était estimée à 13 908 tonnes sur une superficie de 16683 ha avec un rendement moyen de 834 $\mathrm{kg} / \mathrm{ha}$ (ANSD, 2014).

Deux types de mil sont cultivés au Sénégal: le type souna et le type sanio. Le mil souna ou type hâtif est à cycle court (70 à 90 jours) et est presque cultivé sur tout le territoire national (ISRA et al., 2005). Par contre, le type tardif ou mil sanio est de cycle cultural plus long (120 à 150 jours) et est essentiellement cultivé dans les régions naturelles de la Casamance et du Sénégal Oriental (Bamba et al., 2019). Malgré l'importance de cette culture, les rendements restent très faibles en milieu paysan et inférieurs à $750 \mathrm{~kg} / \mathrm{ha}$ (Ndiaye et Sawane, 2015 ; DAPSA, 2016). La faiblesse de ces rendements en grain est essentiellement due à la combinaison de facteurs abiotique (déficit pluviométrique, pauvreté des sols, matériel local rustique et peu productif), biotique (insectes ravageurs, maladies, adventices) et à l'inadéquation des pratiques culturales (Kouakou et al., 2013). Pour améliorer cette situation, la productivité agricole doit passer par une augmentation de la fertilité des sols plutôt que par l'extension des superficies cultivées au détriment des forêts et des terres marginales. Pourtant, l'intérêt d'associer la fumure organique aux engrais minéraux, en vue d'augmenter le niveau de fertilité des sols et le rendement des cultures a été largement démontré par Akanza et Yao, (2011); Akanza et al. (2016); SOMDA et al. (2017) et Zenabou et al., (2014)

C'est dans cette optique que cette étude a été initiée. Elle a pour objectif, d'évaluer les effets de la fertilisation organo-minérale sur la croissance et le rendement en grains du mil sanio afin d'identifier un plan de fumure organo-minérale optimal.

\section{Materiel et methodes}

\subsection{Site d'étude}

L'essai a été conduit pendant l'hivernage 2017 dans la station de Vélingara située en Haute Casamance, au Sud du Sénégal sur des sols ferrugineux tropicaux lessivés (Kanfany, 2009). Le climat est de type sud 
soudanien (Sagna et al., 2012). Le cumul pluviométrique est de $863 \mathrm{~mm}$ en 55 jours de pluie avec une normale climatique (1981-2010) de $856 \mathrm{~mm}$.

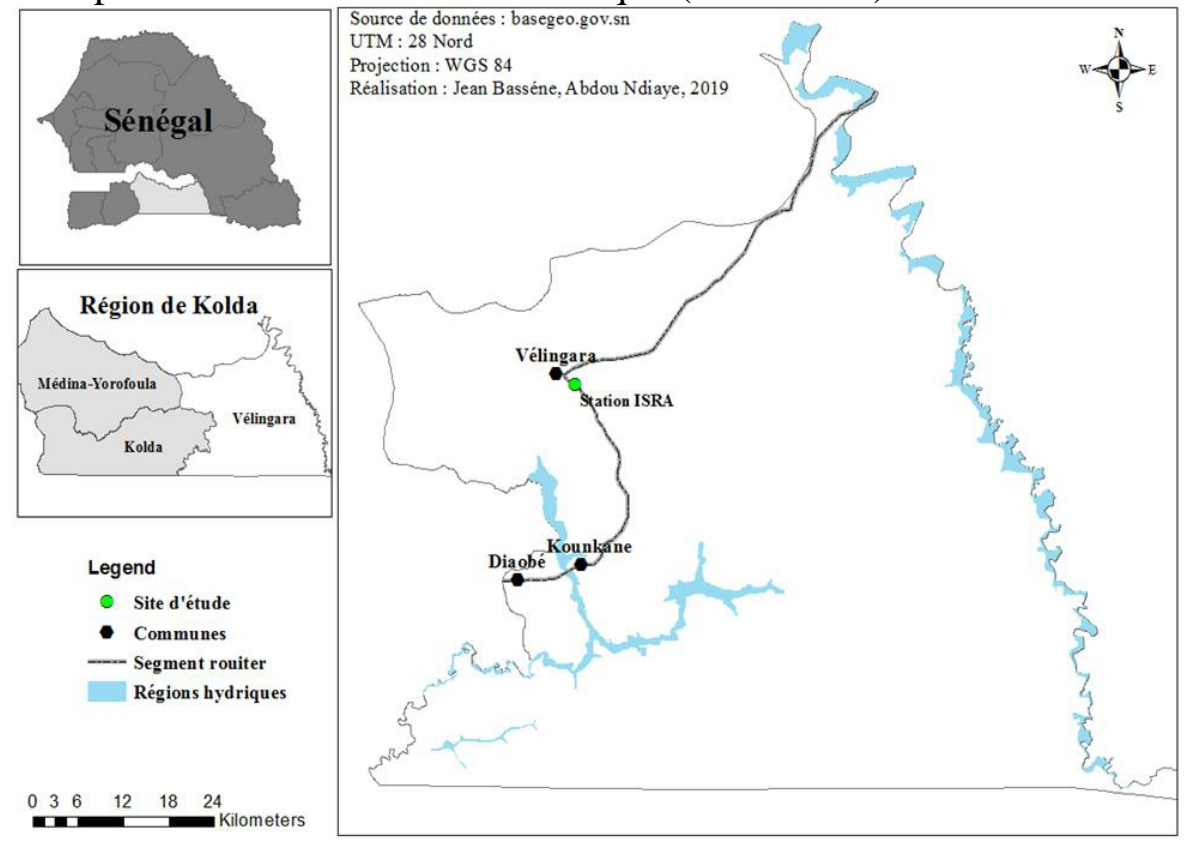

Figure1: Carte de la zone d'étude

\subsection{Matériel végétal}

Le matériel végétal utilisé est un cultivar en provenance de Séfa (Moyenne Casamance, Senégal). Il a un cycle cultural de130 à 150 jours et est caractérisé par des épis aristés. Il est photopériodique et ne fleurit que si la longueur du jour est suffisamment faible (Diouf, 2001).

\subsection{Facteur étudié et dispositif expérimental}

Les facteurs étudiés sont l'amendement organique (AO) et la fertilisation minérale (FM). Le dispositif expérimental est en parcelles subdivisées (split-plot design) avec trois répétitions. Le facteur principal (fertilisation minérale) est placé dans les petites parcelles alors que le facteur secondaire (amendement organique) concerne les grandes parcelles. L'amendement comprend trois niveaux (AO1 : témoin non amendé, $\mathrm{AO} 2: 5$ tonnes par hectare de poudrette d'étable, $\mathrm{AO} 3$ : 10 tonnes par hectare de poudrette d'étable). La fertilisation minérale est constituée de quatre modalités (FM1 : témoin non fertilisé, FM2: $75 \mathrm{~kg} / \mathrm{ha}$ NPK $+50 \mathrm{~kg} / \mathrm{ha}$ Urée (50\% DR), FM3 : $150 \mathrm{~kg} / \mathrm{ha}$ NPK + $100 \mathrm{~kg} / \mathrm{ha}$ Urée (100\% DR), FM4 : $225 \mathrm{~kg} / \mathrm{ha}$ NPK $+150 \mathrm{~kg} /$ ha Urée (150\% DR). L'unité expérimentale est représentée par une parcelle élémentaire de 8 lignes de 7,2 $\mathrm{m}$ de long espacées de $1 \mathrm{~m}$. L'unité 
expérimentale et le carré utile ont une dimension respective de $50,4 \mathrm{~m}^{2}$ (7 $\mathrm{m} * 7,2 \mathrm{~m})$ et $43,2 \mathrm{~m}^{2}\left(6 \mathrm{~m} * 1 \mathrm{~m}^{*} 7,2 \mathrm{~m}\right)$.

$\mathrm{NB}: \mathrm{DR}=$ Dose Recommandée engrais NPK et Urée

\subsection{Conduite de la culture}

Le précédent cultural est une jachère de trois ans. Le semis a été effectué le 14 juillet 2017 sur une parcelle labourée et nivelée. La poudrette d'étable et l'engrais de fond NPK (15-15-15) ont été épandus respectivement un et deux jours après semis et conformément aux modalités. Le démariage à trois plants par poquet a été effectué 15 JAS suivi des apports de l'engrais de couverture (Urée 46\% N) respectivement à la montaison (45 JAS) et à l'épiaison (90 JAS).

Quatre désherbages ont été réalisés (05 JAS ; 17 JAS ; 39 JAS et 55 JAS)

\subsection{Paramètres mesurés}

Les mesures ont porté sur les paramètres morphologiques (hauteur des plantes aux 60 e, 75 e, 90 e et 120 e jours après semis, longueur des entrenœuds et de l'épi, diamètre de l'épi et de la tige au collet) et de production (densité de plants, pourcentage de talles fertiles, nombre d'épis, poids des épis, poids sec biomasse, poids des grains et des mille grains).

\subsection{Analyse statistique}

Les données collectées ont été soumises à l'analyse de variance à l'aide du logiciel Genstat, Discovery édition 4. Les comparaisons multiples des moyennes ont été faites selon le test de Turkey au seuil de $5 \%$

\section{Resultats}

\subsection{La pluviométrie moyenne mensuelle de l'année 2017 à Vélingara}

La figure 1 indique la variation du cumul mensuel de la pluie en 2017 à Vélingara. Elle montre que des quantités importantes d'eau ont été enregistrées au mois d'août avec $492 \mathrm{~mm}$ alors que les mois de septembre et Octobre ont été les moins pluvieux respectivement 43,9 mm et $52.1 \mathrm{~mm}$.

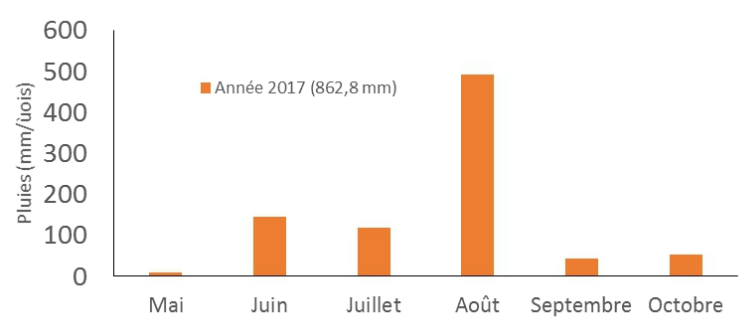

Figure2 : La variation du cumul mensuel de la pluie de l'année 2017 à Vélingara. 


\subsection{Le nombre de tallage et pourcentage de talles fertiles des plants de mil}

La variation du nombre de talles par $\mathrm{m}^{2}$ et le pourcentage de talles fertiles en fonction de l'amendement ainsi que de l'engrais minéral sont consignés dans le tableau 1. L'analyse de ce tableau montre que le tallage n'est pas influencé par l'amendement organique. Par contre la fertilisation minérale a significativement affecté la production de talles $((\mathrm{P}=0,002)$ et le pourcentage de talles fertiles $(\mathrm{P}=0,003)$. La dose recommandée (FM3) et la forte dose (FM4) ont donné statistiquement le nombre de talles $/ \mathrm{m}^{2}$ et le pourcentage les plus élevés.

Tableau 1 : Effet du plan de fumure sur le tallage et le pourcentage de talles fertiles des plantes de mil sanio.

\begin{tabular}{|c|c|c|}
\hline Source de variation & $\begin{array}{c}\text { Nombre de } \\
\text { talles } / \mathrm{m}^{2}\end{array}$ & $\begin{array}{l}\text { Pourcentage de } \\
\text { talles fertiles } \\
\quad(\%)\end{array}$ \\
\hline $\begin{array}{l}\text { Amendement organique (AO, poudrette d'étable) } \\
\text { AO1 }: 0 \text { tonne } \mathrm{ha}^{-1} \text { (témoin non amendé) } \\
\text { AO2 }: 5 \text { tonnes ha }{ }^{-1} \\
\text { AO3 }: 10 \text { tonnes ha }\end{array}$ & $\begin{array}{l}14 \pm 2 \\
13 \pm 2 \\
14 \pm 3\end{array}$ & $\begin{array}{l}70,2 \pm 6,0 \\
68,5 \pm 7,8 \\
70,4 \pm 6,7\end{array}$ \\
\hline $\begin{array}{l}\text { Fertilisation minérale (FM) } \\
\text { FM1 : sans engrais (témoin non fertilisé) } \\
\text { FM2 : } 75 \mathrm{~kg} \mathrm{ha}^{-1} \mathrm{NPK}+50 \mathrm{~kg} \mathrm{ha}^{-1} \text { urée }(50 \%\end{array}$ & $\begin{array}{c}12 \pm 2^{\mathrm{b}} \\
14 \pm 2^{\mathrm{ab}}\end{array}$ & $\begin{array}{l}63,1 \pm 6,0^{c} \\
69,5 \pm 7,2^{c}\end{array}$ \\
\hline $\begin{array}{l}\text { DR) } \\
\text { FM3 : } 150 \mathrm{~kg} \mathrm{ha}^{-1} \mathrm{NPK}+100 \mathrm{~kg} \mathrm{ha}^{-1} \text { urée (DR) } \\
\text { FM4 : } 225 \mathrm{~kg} \mathrm{ha}^{-1} \mathrm{NPK}+150 \mathrm{~kg} \mathrm{ha}^{-1} \text { urée } \\
\text { (150\% DR) }\end{array}$ & $\begin{array}{l}14 \pm 2^{a b} \\
15 \pm 2^{a}\end{array}$ & $\begin{array}{l}72,5 \pm 4,2^{\mathrm{ab}} \\
73,8 \pm 4,0^{\mathrm{a}}\end{array}$ \\
\hline Moyenne \pm Ecart-type $(n=3)$ & $14 \pm 2$ & $69,7 \pm 6,7$ \\
\hline $\begin{array}{l}\text { Coefficient de variation }(\%) \\
\text { AO } \\
\text { FM } \\
\text { AO * FM }\end{array}$ & $\begin{array}{c}10,8 \\
0,774^{\mathrm{ns}} \\
0,002^{*} \\
0,465^{\mathrm{ns}}\end{array}$ & $\begin{array}{c}7,9 \\
0,650^{\mathrm{ns}} \\
0,003^{*} \\
0,419^{\mathrm{ns}}\end{array}$ \\
\hline
\end{tabular}

NPK $=15-15-15 ;$ Urée $=46-0-0 ; \mathrm{DR}=$ dose recommandée $; \mathrm{ns}=$ différence non significative au seuil de $5 \%$.

\subsection{Effet de l'amendement et de la fertilisation minérale sur la taille des plants}

L'évolution de la taille des plantes de mil sanio en fonction de l'amendement et de la fertilisation minérale est représentée dans le tableau 2. Les résultats consignés dans ce tableau montent que l'amendement a significativement $(P=0,043)$ influé la hauteur des plantes de mil sanio. A 90 et 120 JAS, les parcelles fumées avec 5 ou 10 tonnes par hectare de poudrette d'étable ont donné la taille des plantes les plus hautes par rapport au témoin. De même, la taille des plantes (tableau 2) est aussi significativement $(\mathrm{P}<0,05)$ influencée par l'augmentation des apports d'engrais minéral. Les plantes les plus développées sont observées dans les parcelles fertilisées à la dose 
recommandée (FM3) et à la forte dose (FM4)). A $120 \mathrm{JAS}$, les traitements les doses FM3 et FM4 ont obtenus une augmentation de 13,7 et de 16,4\% de la taille des plantes respectivement par rapport au témoin FM1

Tableau 2: Effet de l'amendement et de l'engrais minéral sur la taille des plantes de mi sanio à 60 , à 75 , à 90 et à 120 JAS.

\begin{tabular}{|c|c|c|c|c|}
\hline \multirow{2}{*}{ Source de variation } & \multicolumn{4}{|c|}{ Hauteur des plantes $(\mathrm{cm})$} \\
\hline & $60 \mathrm{JAS}$ & 75 JAS & $90 \mathrm{JAS}$ & $120 \mathrm{JAS}$ \\
\hline \multicolumn{5}{|l|}{$\begin{array}{l}\text { Amendement organique (AO, } \\
\text { poudrette d'étable) }\end{array}$} \\
\hline $\begin{array}{l}\text { AO1 : } 0 \text { tonne ha }{ }^{-1} \text { (témoin } \\
\text { non amendé) }\end{array}$ & $73,9 \pm 14,7$ & $158,5 \pm 27,3$ & $234,3 \pm 15,0^{b}$ & $250,9 \pm 18,2^{b}$ \\
\hline $\mathrm{AO} 2: 5$ tonnes ha ${ }^{-1}$ & $82,3 \pm 17,8$ & $171,8 \pm 25,9$ & $247,1 \pm 18,7^{\mathrm{ab}}$ & $266,0 \pm 27,2$ \\
\hline AO3 $: 10$ tonnes $\mathrm{ha}^{-1}$ & $94,3 \pm 14,0$ & $193,8 \pm 19,3$ & $267,4 \pm 17,9^{\mathrm{a}}$ & $291,1 \pm 21,4^{\mathrm{a}}$ \\
\hline Fertilisation minérale (FM) & & & & \\
\hline $\begin{array}{l}\text { FM1 : sans engrais (témoin } \\
\text { non fertilisé) }\end{array}$ & $73,2 \pm 20,2^{b}$ & $150,6 \pm 23,7^{b}$ & $235,6 \pm 23,1^{b}$ & $244,1 \pm 24,0^{b}$ \\
\hline $\begin{array}{l}\text { FM2 : } 75 \mathrm{~kg} \mathrm{ha}^{-1} \mathrm{NPK}+50 \\
\mathrm{~kg} \mathrm{ha}^{-1} \text { urée }(50 \% \text { DR })\end{array}$ & $84,1 \pm 12,3^{a b}$ & $173,1 \pm 17,7^{b}$ & $247,5 \pm 14,2^{b}$ & $271,5 \pm 15,4^{b}$ \\
\hline $\begin{array}{l}\text { FM3 : } 150 \mathrm{~kg} \mathrm{ha}^{-1} \mathrm{NPK}+ \\
100 \mathrm{~kg} \mathrm{ha}^{-1} \text { urée (DR) }\end{array}$ & $92,0 \pm 18,8^{a b}$ & $190,6 \pm 27,0^{b}$ & $260,9 \pm 23,5^{\mathrm{ab}}$ & $277,5 \pm 32,5^{b}$ \\
\hline $\begin{array}{l}\text { FM4 : } 225 \mathrm{~kg} \mathrm{ha}^{-1} \mathrm{NPK}+ \\
150 \mathrm{~kg} \mathrm{ha}^{-1} \text { urée (150\% DR) }\end{array}$ & $84,9 \pm 14,0^{\mathrm{a}}$ & $184,6 \pm 27,6^{\mathrm{a}}$ & $254,4 \pm 19,9^{\mathrm{a}}$ & $284,2 \pm 20,6^{\mathrm{a}}$ \\
\hline Moyenne \pm Ecart-type $(\mathrm{n}=3)$ & $83,5 \pm 17,2$ & $174,7 \pm 28,0$ & $249,6 \pm 21,8$ & $269,3 \pm 27,6$ \\
\hline Coefficient de variation (\%) & 11,6 & 7,8 & 5 & 5,5 \\
\hline Probabilité et signification & & & & \\
\hline $\mathrm{AO}$ & $0,294^{\mathrm{ns}}$ & $0,129^{\mathrm{ns}}$ & $0,043^{*}$ & $0,017^{*}$ \\
\hline FM & $0,006^{*}$ & $0,001^{* *}$ & $0,003^{*}$ & $0,001^{* *}$ \\
\hline $\mathrm{AO} * \mathrm{FM}$ & $0,073^{\mathrm{ns}}$ & $0,122^{\mathrm{ns}}$ & $0,279^{\mathrm{ns}}$ & $0,181^{\mathrm{ns}}$ \\
\hline $\begin{array}{l}\text { Plus petite différence } \\
\text { significative }\end{array}$ & 30,63 & 37,47 & 26,33 & 27,45 \\
\hline
\end{tabular}

NPK $=15-15-15 ;$ Urée $=46-0-0 ; \mathrm{DR}=$ dose recommandée $; \mathrm{ns}=$ différence non significative au seuil de $5 \% ; *$ différence significative au seuil de $1 \% ; * *=$ différence hautement significative au seuil de $0,1 \%$; JAS = jours après semis

\subsection{Effet de l'amendement et de la fertilisation minérale sur la croissance radiale et l'élongation de la tige.}

Le diamètre de la tige au collet n'est pas statistiquement influencé par les apports organo-minéraux (tableau 3). En moyenne le diamètre de la tige est de $1,4 \pm 0,1 \mathrm{~cm}$ indépendamment du niveau de l'amendement et de l'engrais minéral.

L'engrais organique n'a pas eu d'effet significatif sur l'élongation de la tige. Par contre, la fertilisation minérale a significativement $(P=0,002)$ affecté la longueur des entre nœuds. En effet, les tiges les plus développées ont été notées dans les parcelles fertilisées au moins aux doses recommandées, soit plus de 4,2-5,4\% en comparaison au témoin non fertilisé. 
Tableau 3: Influence de la matière organique et de l'engrais minéral sur le diamètre de la tige et la distance des entre noeuds.

\begin{tabular}{|c|c|c|}
\hline Source de variation & $\begin{array}{l}\text { Diamètre de la } \\
\text { tige au collet } \\
(\mathrm{cm})\end{array}$ & $\begin{array}{l}\text { Distance entre } \\
\text { nœuds }(\mathrm{cm})\end{array}$ \\
\hline \multicolumn{3}{|l|}{ Amendement organique (AO, poudrette d'étable) } \\
\hline AO1 : 0 tonne ha ${ }^{-1}$ (témoin non amendé) & $1,3 \pm 0,1$ & $17,2 \pm 0,7$ \\
\hline $\mathrm{AO} 2: 5$ tonnes $\mathrm{ha}^{-1}$ & $1,4 \pm 0,1$ & $17,1 \pm 0,8$ \\
\hline $\mathrm{AO} 3: 10$ tonnes ha ${ }^{-1}$ & $1,5 \pm 0,1$ & $17,6 \pm 1.0$ \\
\hline \multicolumn{3}{|l|}{ Fertilisation minérale (FM) } \\
\hline FM1 : sans engrais (témoin non fertilisé) & $1,4 \pm 0,2$ & $16,8 \pm 0,9^{\mathrm{c}}$ \\
\hline FM2 : $75 \mathrm{~kg} \mathrm{ha}^{-1} \mathrm{NPK}+50 \mathrm{~kg} \mathrm{ha}^{-1}$ urée (50\% DR) & $1,4 \pm 0,1$ & $17,3 \pm 0,7^{\mathrm{bc}}$ \\
\hline FM3 : $150 \mathrm{~kg} \mathrm{ha}^{-1} \mathrm{NPK}+100 \mathrm{~kg} \mathrm{ha}^{-1}$ urée (DR) & $1,5 \pm 0,2$ & $17,5 \pm 0,7^{\mathrm{a}}$ \\
\hline FM4 : $225 \mathrm{~kg} \mathrm{ha}^{-1} \mathrm{NPK}+150 \mathrm{~kg} \mathrm{ha}^{-1}$ urée $(150 \%$ & $1,4 \pm 0,1$ & $17,7 \pm 1.0^{\mathrm{a}}$ \\
\hline \multicolumn{3}{|l|}{ DR) } \\
\hline Moyenne \pm Ecart-type $(n=3)$ & $1,4 \pm 0,1$ & $17,3 \pm 0,8$ \\
\hline Coefficient de variation $(\%)$ & 8,5 & 2,5 \\
\hline \multicolumn{3}{|l|}{ Probabilité et signification } \\
\hline $\mathrm{AO}$ & $0,100^{\mathrm{ns}}$ & $0,528^{\mathrm{ns}}$ \\
\hline FM & $0,510^{\mathrm{ns}}$ & $0,002 *$ \\
\hline $\mathrm{AO} * \mathrm{FM}$ & $0,718^{\mathrm{ns}}$ & $0,404^{\mathrm{ns}}$ \\
\hline
\end{tabular}

NPK $=15-15-15 ;$ Urée $=46-0-0 ; \mathrm{DR}=$ dose recommandée $; \mathrm{ns}=$ différence non significative au seuil de $5 \%$.

\subsection{Effet de l'amendement et de la fertilisation minérale sur les caractéristiques de l'épi}

Les différentes doses de matière organique et/ou de l'engrais minéral n'ont pas eu d'effet significatif sur le diamètre et la longueur des épis de mil sanio (tableau4). En moyenne, le diamètre et la longueur des épis de mil sanio sont respectivement de $1,8 \pm 0,11$ et $33,4 \pm 2,1 \mathrm{~cm}$ quelle que soit la modalité des facteurs appliqués.

Tableau 4 : Effet de l'amendement et de l'engrais minéral sur le diamètre et la longueur de l'épi.

\begin{tabular}{|c|c|c|}
\hline Source de variation & $\begin{array}{l}\text { Diamètre de } \\
\text { l'épi }(\mathrm{cm})\end{array}$ & $\begin{array}{l}\text { Longueur de } \\
\text { l'épi }(\mathrm{cm})\end{array}$ \\
\hline \multicolumn{3}{|l|}{ Amendement organique (AO, poudrette d'étable) } \\
\hline AO1 : 0 tonne ha ${ }^{-1}$ (témoin non amendé) & $1,9 \pm 0,2$ & $33,2 \pm 1,9$ \\
\hline $\mathrm{AO} 2: 5$ tonnes $\mathrm{ha}^{-1}$ & $1,8 \pm 0,1$ & $34,0 \pm 2,5$ \\
\hline AO3 $: 10$ tonnes ha $^{-1}$ & $1,8 \pm 0,1$ & $33,0 \pm 2,0$ \\
\hline \multicolumn{3}{|l|}{ Fertilisation minérale (FM) } \\
\hline FM1 : sans engrais (témoin non fertilisé) & $1,8 \pm 0,1$ & $34,3 \pm 1,7$ \\
\hline FM2 : $75 \mathrm{~kg} \mathrm{ha}^{-1} \mathrm{NPK}+50 \mathrm{~kg} \mathrm{ha}^{-1}$ urée (50\% DR) & $1,9 \pm 0,1$ & $33,5 \pm 2,4$ \\
\hline FM3 : $150 \mathrm{~kg} \mathrm{ha}^{-1} \mathrm{NPK}+100 \mathrm{~kg} \mathrm{ha}^{-1}$ urée (DR) & $1,8 \pm 0,1$ & $32,3 \pm 2,0$ \\
\hline FM4 : $225 \mathrm{~kg} \mathrm{ha}^{-1} \mathrm{NPK}+150 \mathrm{~kg} \mathrm{ha}^{-1}$ urée $(150 \%$ & $1,8 \pm 0,1$ & $33,5 \pm 2,2$ \\
\hline DR) & & \\
\hline
\end{tabular}




\begin{tabular}{lll}
\hline Moyenne \pm Ecart-type $(\mathrm{n}=3)$ & $1,8 \pm 0,1$ & $33,4 \pm 2,1$ \\
\hline $\begin{array}{l}\text { Coefficient de variation }(\%) \\
\text { Probabilité et signification }\end{array}$ & 6,5 & 6,6 \\
AO & $0,311^{\mathrm{ns}}$ & $0,384^{\mathrm{ns}}$ \\
FM & $0,170^{\mathrm{ns}}$ & $0,322^{\mathrm{ns}}$ \\
AO $*$ FM & $0,949^{\mathrm{ns}}$ & $0,607^{\mathrm{ns}}$ \\
\hline
\end{tabular}

NPK $=15-15-15 ;$ Urée $=46-0-0 ; \mathrm{DR}=$ dose recommandée $; \mathrm{ns}=$ différence non significative au seuil de $5 \%$.

\subsection{Effet de l'amendement et de la fertilisation minérale sur le nombre d'épi, le poids d'épis et le poids des grains}

Le tableau 5 montre que l'amendement organique n'a pas significativement affecté le nombre d'épis, le poids d'épis et le poids des grains du mil sanio. Par contre la fertilisation a hautement influencé ces paramètres $(\mathrm{P}=0,001)$. L'analyse du tableau 5 montre une augmentation par rapport au témoin du nombre d'épis, du poids d'épis et du poids des grains par hectare suite à l'apport de 10 tonnes de poudrette d'étable par hectare. Le traitement A3 (10 tonnes de poudrette par hectare) a permis d'accroitre respectivement de $10,3 \%$ le nombre d'épis, de $37,2 \%$ le poids d'épis et de $59,5 \%$ le poids grain par rapport aux parcelles non amendées.

Les performances en nombre d'épis, en poids d'épis et en poids grains sont obtenues dans les parcelles fertilisées à la dose recommandée. Les traitements FM3 et FM4 ont permis de noter dans l'ordre par rapport au témoin non fertilisé (FM1) un gain de 47,9 et $66,1 \%$ pour le nombre d'épis, de 41,1 et $71,4 \%$ pour le poids d'épis et de 29,3 et $62,3 \%$ pour le poids des grains.

Tableau 5 : Effet de l'amendement et de la fertilisation minérale sur les composantes du rendement

\begin{tabular}{|c|c|c|c|}
\hline Source de variation & Nombre de l'épi ha ${ }^{-1}$ & $\begin{array}{l}\text { Poids d'épi (kg } \\
\left.\text { ha }^{-1}\right)\end{array}$ & $\begin{array}{c}\text { Rendement grains } \\
\left(\mathrm{kg} \mathrm{ha}^{-1}\right)\end{array}$ \\
\hline \multicolumn{4}{|l|}{$\begin{array}{l}\text { Amendement organique (AO, } \\
\text { poudrette d'étable) }\end{array}$} \\
\hline $\begin{array}{l}\text { AO1 : } 0 \text { tonne } \mathrm{ha}^{-1} \text { (témoin } \\
\text { non amendé) }\end{array}$ & $69946 \pm 13507$ & $1179 \pm 332$ & $513 \pm 291$ \\
\hline $\mathrm{AO} 2: 5$ tonnes ha- ${ }^{-1}$ & $69579 \pm 15700$ & $1237 \pm 415$ & $585 \pm 260$ \\
\hline AO3 : 10 tonnes ha ${ }^{-1}$ & $77141 \pm 15191$ & $1618 \pm 307$ & $818 \pm 166$ \\
\hline \multicolumn{4}{|l|}{ Fertilisation minérale (FM) } \\
\hline $\begin{array}{l}\text { FM1 : sans engrais (témoin } \\
\text { non fertilisé) }\end{array}$ & $52803 \pm 6142^{\mathrm{d}}$ & $983 \pm 304^{c}$ & $485 \pm 209^{c}$ \\
\hline $\begin{array}{l}\text { FM2 : } 75 \mathrm{~kg} \mathrm{ha}^{-1} \mathrm{NPK}+50 \\
\mathrm{~kg} \mathrm{ha}^{-1} \text { urée }(50 \% \text { DR })\end{array}$ & $70293 \pm 8582^{c}$ & $1335 \pm 339^{b}$ & $655 \pm 283^{b}$ \\
\hline $\begin{array}{l}\text { FM3 : } 150 \mathrm{~kg} \mathrm{ha}^{-1} \mathrm{NPK}+ \\
100 \mathrm{~kg} \mathrm{ha}^{-1} \text { urée (DR) }\end{array}$ & $78112 \pm 5874^{b}$ & $1377 \pm 330^{b}$ & $627 \pm 273^{b}$ \\
\hline $\begin{array}{c}\text { FM4 : } 225 \mathrm{~kg} \mathrm{ha}^{-1} \mathrm{NPK}+ \\
150 \mathrm{~kg} \mathrm{ha}^{-1} \text { urée }(150 \% \mathrm{DR})\end{array}$ & $87680 \pm 8915^{\mathrm{a}}$ & $1685 \pm 307^{a}$ & $787 \pm 271^{\mathrm{a}}$ \\
\hline Moyenne \pm Ecart-type $(\mathrm{n}=3)$ & $72222 \pm 14826$ & $1345 \pm 397$ & $639 \pm 272$ \\
\hline
\end{tabular}




\begin{tabular}{lccc}
\hline $\begin{array}{l}\text { Coefficient de variation }(\%) \\
\text { Probabilité et signification }\end{array}$ & 7,4 & 8,6 & 13,0 \\
AO & $0,254^{\mathrm{ns}}$ & $0,054^{\mathrm{ns}}$ & $0,140^{\mathrm{ns}}$ \\
FM & $0,001^{* *}$ & $0,001^{* *}$ & $0,001^{* *}$ \\
AO $*$ FM & $0,234^{\mathrm{ns}}$ & $0,195^{\mathrm{ns}}$ & $0,420^{\mathrm{ns}}$ \\
\hline
\end{tabular}

\subsection{Effet de la fertilisation organo-minérale sur la biomasse aérienne sèche (tiges+feuilles) et le poids 1000 grains}

L'évolution de la biomasse aérienne en fonction de l'amendement organique et de la fertilisation minérale est consignée dans le tableau 6. L'analyse de ce tableau révèle que les doses d'amendement organique n'ont pas eu un effet significatif sur la biomasse aérienne (tige+feuille) du mil sanio. Par contre, la biomasse aérienne est très significativement $(P=0,001)$ affectée par les doses d'engrais minéral. Les valeurs de biomasse tiges les plus élevées sont enregistrées dans les parcelles ayant reçu de l'engrais minéral. En effet les traitements $50 \%$ DR, $100 \%$ DR et $150 \%$ DR ont permis de noter respectivement un gain de $26 \%$, de $32 \%$ et de $56 \%$ de biomasse tige aérienne par rapport au témoin non fertilisé. En ce qui concerne le poids 1000 grains, il n'est ni affecté par les apports de matière organique ni par les doses d'engrais minéral. En moyenne, le poids des 1000 grains est de 5,3 grammes.

Tableau 6: Effet de l'amendement et de l'engrais minéral sur la biomasse aérienne (tiges) de mil sanio

\begin{tabular}{|c|c|c|}
\hline Source de variation & $\begin{array}{l}\text { Rendement de la } \\
\text { Biomasse aérienne } \\
\text { sèche }\left(\mathrm{kg} \mathrm{ha}^{-1}\right)\end{array}$ & $\begin{array}{c}\text { Poids } \\
1000 \\
\text { grains }(\mathrm{g}) \\
\end{array}$ \\
\hline \multicolumn{3}{|l|}{ Amendement organique (AO, poudrette d'étable) } \\
\hline AO1 : 0 tonne ha ${ }^{-1}$ (témoin non amendé) & $5983 \pm 1805$ & $5,3 \pm 0,3$ \\
\hline $\mathrm{AO} 2: 5$ tonnes $\mathrm{ha}^{-1}$ & $6876 \pm 1599$ & $5,4 \pm 0,2$ \\
\hline AO3 $: 10$ tonnes ha ${ }^{-1}$ & $8631 \pm 1423$ & $5,3 \pm 0,3$ \\
\hline \multicolumn{3}{|l|}{ Fertilisation minérale (FM) } \\
\hline FM1 : sans engrais (témoin non fertilisé) & $5576 \pm 1822^{c}$ & $5,4 \pm 0,3$ \\
\hline FM2 : $75 \mathrm{~kg} \mathrm{ha}^{-1} \mathrm{NPK}+50 \mathrm{~kg} \mathrm{ha}^{-1}$ urée $(50 \%$ & $7029 \pm 1301^{b c}$ & $5,2 \pm 0,3$ \\
\hline \multicolumn{3}{|l|}{ DR) } \\
\hline FM3 : $150 \mathrm{~kg} \mathrm{ha}^{-1} \mathrm{NPK}+100 \mathrm{~kg} \mathrm{ha}^{-1}$ urée (DR) & $7376 \pm 1693^{b}$ & $5,3 \pm 0,2$ \\
\hline $\begin{array}{l}\text { FM4 : } 225 \mathrm{~kg} \mathrm{ha}^{-1} \mathrm{NPK}+150 \mathrm{~kg} \mathrm{ha}^{-1} \text { urée } \\
\text { (150\% DR) }\end{array}$ & $8672 \pm 1697^{a}$ & $5,3 \pm 0,3$ \\
\hline Moyenne \pm Ecart-type $(\mathrm{n}=3)$ & $7163 \pm 1926$ & $5,3 \pm 0,3$ \\
\hline Coefficient de variation $(\%)$ & 14.9 & 3,0 \\
\hline \multicolumn{3}{|l|}{ Probabilité et signification } \\
\hline $\mathrm{AO}$ & $0,082^{\mathrm{ns}}$ & $0,614^{\mathrm{ns}}$ \\
\hline FM & $0,001^{* *}$ & $0,468^{\mathrm{ns}}$ \\
\hline $\mathrm{AO} * \mathrm{FM}$ & $0,840^{\mathrm{ns}}$ & $0,460^{\mathrm{ns}}$ \\
\hline
\end{tabular}

NPK $=15-15-15 ;$ Urée $=46-0-0 ; \mathrm{DR}=$ dose recommandée $; \mathrm{ns}=$ différence non significative au seuil de $5 \% ; * *=$ différence hautement significative au seuil de $0,1 \%$. 


\section{Disscusion}

Durant le cycle cultural, la pluviométrie totale enregistrée dans le site était de $863 \mathrm{~mm}$ répartie en 55 jours de pluie. Une pause pluviométrie de 25 jours était notée au mois de Septembre. Cette poche de sécheresse a coïncidé avec la phase de reproduction. Or, pendant cette période le mil a besoin d'une quantité importante d'eau ce qui impact négativement sur la productivité (Diouf, 1990).

Les résultats ont montré que l'amendement organique a eu un effet significatif sur la hauteur des plantes à partir de 90 et 120 jours après semis (JAS), tandis que la fertilisation minérale a eu un effet positif dès $60 \mathrm{JAS}$. Ce retard d'effet de l'amendement par rapport à l'engrais minéral peut s'expliquer par la vitesse de minéralisation des amendements organiques. En effet les constituants des amendements organiques ne sont pas directement disponibles, ils doivent d'abord être minéralisés (Asdrubal et al., 2006 et Segnou et al., 2012). Selon Etter, 2017, le fumier de volaille et les farines animales sont rapidement minéralisés (dès les premiers mois après l'épandage) alors que le fumier de bovins nécessite un temps important . L'augmentation de la taille de la plante a été notée suite à une fertilisation organo-minérale. Des résultats similaires ont été trouvés par Cissé (1988) qui a montré un effet positif de la fertilisation organo-minérale sur la hauteur des plantes de mil, sur le sorgho par Somda et al (2017) et sur le fonio par Gueye (2016). Cette augmentation peut s'expliquer par le fait que l'application des amendements organiques et d'engrais minéraux rend plus disponible les éléments de croissance des plantes tels que le phosphore comme l'indique Somda et al. (2017). Par ailleurs, la fertilisation a eu aussi des effets significatifs sur la distance des entre-nœuds des plantes et sur le pourcentage de talles fertiles. Ce résultat sur le tallage productif est confirmé par les travaux de Eldin (1990). Ce dernier a montré une augmentation du nombre de talles du mil suite à une fertilisation minérale.

Pour les paramètres du rendement, les résultats ont montré que seule l'application des doses de l'engrais minéral a eu un effet positif sur les composantes du rendement de mil sanio. En effet, le nombre d'épis, le poids d'épis, le rendement en grains et la biomasse des tiges aériennes ont augmenté suite à une application croissante des doses d'engrais minéral. L'application de $150 \%$ de la dose recommandée d'engrais minéral a donné le meilleur résultat. Ces résultats sont en phase avec ceux de Eldin (1990) qui a montré que le nombre de grains par épi et le poids du grain du mil montrent une excellente réponse à la fertilisation minérale. Cependant ces résultats ne sont pas en phase avec ceux trouvés par Badiane (1986). Cet auteur a enregistré une très grande réponse sur le poids d'épis, le poids tiges et le poids grains chez une variété de mil Souna à la dose 04 tonnes/ha de fumier. En effet cette dose a induit une augmentation de $1005 \mathrm{~kg}$ du poids d'épis par rapport au témoin non amendé, de $2031 \mathrm{~kg}$ du poids tige et de $706 \mathrm{~kg}$ du poids grains. 
Cela pourrait s'expliquer par l'effet du cultivar mais aussi par le fait que son étude a été réalisée dans une rotation arachide/mil.

L'interaction entre l'amendement et la fertilisation minérale n'a pas d'effet tant pour les paramètres de croissance que sur les paramètres de production du mil sanio. Ces résultats sont en accord avec ceux trouvés par Dembele (1994) qui a montré que l'effet d'interaction entre l'amendement organique et d'engrais minéral est absent en première année d'application. Par contre, ces résultats ne vont pas dans le même sens avec ceux trouvés par Akanza et Yao (2011), INRAN (2013) et Somda (2017). Ces auteurs ont montré des accroissements importants par rapport au témoin suite à l'apport de fiente de volaille et engrais minéral sur la hauteur, et sur le rendement du sorgho, du mil et en racines des tubercules de manioc. Ce manque d'effet d'interaction pourrait être lié à la qualité du fumier appliqué comme l'indiquent Kaho et al. (2011). En effet, les fumiers organiques n'ont pas les mêmes valeurs nutritives en éléments fertilisants (Siboukeur, 2013). Il montre que la fiente de volaille est beaucoup plus riche en éléments fertilisants comparé aux autres fumiers organiques. En plus, Ndour et al. (2016) et Equiterre (2009) ont montré que la fiente de volaille est à peu près six fois plus riche en azote que celui de bovin.

Pour la variation du poids des 1000 grains, les résultats ont montré qu'il n'y a pas une différence significative entre les traitements. Le poids varie entre 5,08 et 5,50 g. Ces résultats sont similaires à ceux de Diouf (2001) sur le mil. Par contre, ce poids est inférieur à celui trouvé par les travaux de l'ISRA en 1974 (6,13 à 6,92 g) et Diouf et al. en 2014 (10 à 12g). Cela peut s'expliquer par un mauvais remplissage des grains lié à la pause pluviométrique ( 25 jours) observée pendant la phase de maturation. Cette absence de différence entre les traitements, peut s'expliquer par le fait que nos semences proviennent de la même variété. En effet le poids 1000 grains fait partie des principaux caractères physiques qui permettent de discriminer les variétés (Ouattara et al., 2015). Ainsi, selon ce dernier, le poids varie entre 9,2 et $13,7 \mathrm{~g}$ pour le mil. Des résultats similaires ont été trouvés par Cheik et al. (2006) et Békoye (2014). Le poids moyen d'un grain dépend de la phase de remplissage des grains (Rouw et al., 2004).

\section{Conclusion}

Cette étude menée à la station de recherche de l'ISRA à Vélingara a permis de mettre en évidence l'effet de la fertilisation organo-minérale sur les paramètres de croissance et de production du mil sanio en Haute Casamance. Les résultats ont montré que l'interaction entre l'amendement organique et la fertilisation minérale n'est pas significative sur l'ensemble des paramètres mesurés. Par contre les doses d'amendement organique ont eu un effet positif sur la taille des plantes de mil sanio à 90 et $120 \mathrm{JAS}$. La dose de 10 t/ha a 
donné les hauteurs de plantes les plus longues $(267 \mathrm{~cm}$ à 90 JAS et $291 \mathrm{~cm}$ à 120 JAS). Ces doses n'ont pas d'effet significatifs sur les paramètres du rendement, néanmoins la dose 10 tonnes/ha de poudrette a induit une augmentation du poids grains de $59 \%$ par rapport au témoin non amendé. La fertilisation minérale a eu des effets significatifs sur les paramètres de croissance (la hauteur, la distance entre nœuds) et de production (le tallage productif, le nombre d'épis, le poids d'épis, le rendement en grains et la biomasse des tiges aériennes). L'apport de 50\% de la dose d'engrais minéral vulgarisée a favorisé un accroissement de $37 \%$ du rendement en grains par rapport au témoin non fertilisé.

En guise de perspective, il serait nécessaire de reconduire l'essai en station et en milieu paysans pour confirmer ou infirmer les résultats, de tester d'autres types de fumiers comme sources de fertilisants organiques (la fiente de volaille ou la farine d'origine animale) et de procéder au sélection variétale en créant des variétés moins sensible à la sècheresse.

\section{Conflit d'interets}

Les auteurs déclarent sur l'honneur l'absence de tout conflit d'intérêt.

\section{Contributions Des Auteurs}

AN a été l'investigateur principal et a réalisé les travaux de terrain. $\mathrm{AN}, \mathrm{BB}, \mathrm{MG}$, et $\mathrm{ON}$ ont contribué à la rédaction du protocole, à l'acquisition, l'analyse et à l'interprétation des données, à la rédaction du manuscrit. ON, $\mathrm{BB}, \mathrm{MG}$, et $\mathrm{OS}$ ont contribué à la conception et supervision des travaux, à la révision du protocole et du manuscrit.

\section{Remerciements}

Les auteurs remercient M Moussa Kandé le technicien ISRA au PAPEM de Vélingara et sa femme Diabou Baldé pour l'appui.

\section{References:}

1. Akanza K. P., Sanogo S., N’Da H. A., 2016. Influence combinée des fumures organique et minérale sur la nutrition et le rendement du maïs : Impact sur le diagnostic des carences du sol. TROPICULTURA. 34 (2) 208- 220.

2. Akanza K.P., Yayo K. A., 2011. Fertilisation organo-minérale du manioc (Manihot esculenta Crantz) et diagnostic des carences du sol. Journal of Applied Biosciences 46: 3163-3172, 10 pages.

3. ANSD. 2014. Bulletin mensuel des statistiques économiques, Décembre 2014. ISSN 0850-1467.

4. ANSD, 2015. Situation économique et sociale du Sénégal en 2012. 09 pages. 
5. Asdrubal M., Sylvie D., Charonnat C., Denys F., Fresse J.C., Thomas J .M., 2006. Fertilisation et amendements. Educagri éditions, 131 pages.

6. Badiane A. N., 1986. Courbe de réponse A de doses croissantes de fumier (thilmakha) et Essai travail du sol (sole III Nord- Bambey).

7. BAMBA B., GUEYE M., NGOM D., KA S. L., DIOP B. et KANFANY G., 2019.

8. Caractérisation des pratiques locales du mil Sanio [Pennisetum glaucum (L.) R. Br] en zone soudanienne humide au Sénégal. In : Int. J. Biol. Chem. Sci. 13(2): 1054-1063, April 2019, 10 pagesBékoye B.M., 2014. Caractérisation physico chimique et technologique des variétés de mil pennisetum glaucum (1.) R. Br.) Ouest africaines. In: European Scientific Journal.; vol.10, No.30 ISSN: 1857 - 7881 (Print) e - ISSN 1857- 7431, 10 pages.

9. Cheik A.T.O., Savadogo A., Bayane Y. et Traore S. A., 2006. A Comparative Study on Nutritional and Technological Quality of Fourteen (14) Cultivars of Pearl Millets [Pennisetum glaucum (L) Leeke] in Burkina Faso. In: Pakistan Journal of Nutrition 5 (6): 512 521, ISSN 1680-5194, (C Asian Network for Scientific Information, 10 pages.

10. Cisse L., 1988. Influence d'apports de matière organique sur la culture de mil et d'arachide sur un sol sableux du Nord Sénégal. II. Développement des plantes et mobilisations minérales. EDP Sciences, 1988, 8 (5), pp.411-417. <hal-00885118>, 08 pages.

11. DAPSA., 2016. Rapport de performance 2015. Ministère de l'agriculture et de l'équipement rural, Direction de l'Analyse de la Prévision et des Statistiques Agricoles, 52 pages.

12. Dembele I., 1994. Production et utilisation de la fumure organique. Fiche synthétique d'information, 19 pages.

13. Diouf M., 1990. Analyse de l'élaboration du rendement du mil (Pennisetum tryphoides Stapf et Hubb) mise au point d'une méthode de diagnostic en parcelle paysanne. Thèse de doctorat, Institut National Agronomique. Paris-Grignon, 248 pages.

14. Diouf O., 2001. La culture du mil (Pennisetum glaucum (L.) R. Br.) en zone siemi-aride : bases agrophysiologiques justificatives d'une fertilisation azotée. Mémoire de titularisation, Institut Sénégalais de Recherches Agricoles (ISRA), 75 pages.

15. Diouf N., Ndiaye O., Mar A., et Diallo M., 2014. Le mil. Exposé, 14 pages.

16. Eldin O., 1990. Croissance et developpement du mil (Pennisetum typhoides) sous deux conditions de fumure minérale. Rapport de stage, université de Niamey Niger, 53 pages. 
17. Equiterre, 2009. Les amendements organiques : fumiers et composts. Module 7, chapitre 12, 19 pages.

18. Etter A., 2017. Engrais organiques: rendement et qualité. Revu UFA 10/ 2017, 2 pages.

19. FAO, 2016. Sénégal: la FAO confirme une production recorde de 2.27 tonnes de céréales en 2015.https//actuprime.com/Sénégal-la Fao confirme -une - production, consulté le 10/05/2018.

20. FAO, 2017. FAOSTAT (2003). http://faostat3.fao.org/download/Q/QC/F/ Consulté en mai 2018

21. Gueye M., 2016. Amélioration des techniques de semis, de fertilisation et de récolte du fonio blanc (Digitaria exilis Stapf ; Poaceae) au Sénégal Oriental et en Casamance (Sénégal). Thèse doctorant, faculté des sciences et techniques, université cheikh Anta Diop de Dakar, 137 pages.

22. INRAN et DAH, 2013. Poultry manure and inorganic fertilizer to improve pearl millet yield in Niger. In: African Journal of Plant Science, Vol. 7(5), pp. 162-169, 08 pages.

23. ISRA, 1974. Amélioration Des Mils Au Sénégal. Rapport général d'activité, Vol. 1. Amélioration variétale, 208 pages.

24. ISRA, ITA, CIRAD. 2005. Bilan de la recherche agricole et agroalimentaire au Sénégal. Institut sénégalais de recherches agricoles, p 524.

25. Kanfany G., 2009. Effets de la fertilisation organo-minérale sur la croissance et rendement du fonio. Mémoire de fin d'étude d'école nationale supérieure d'agriculture de Thiès/Sénégal.

26. Kouakou PK, Muller B. Guissé A. Yao RN, Fofana A, Cissé N. 2013. Étude et prise en compte en modélisation de l'effet de la latitude sur la réponse à la photopériode chez divers génotypes de mil (Pennisetum glaucum (L.) R. Br.) du Sénégal. J. Appl. Biosci., 67: 5289-5301. DOI : www.m.elewa.org/JABS/2013/67/Abstract12-kouakou.html.

27. Kaho F., Yemefack M., Feujio P., Teguefouet et J.C Tchantchaouang J.C., 2011. Effet combiné des feuilles de Tithonia diversifolia et des engrais inorganiques sur les rendements du maïs et les propriétés d'un sol ferralitique au Centre Cameroun. In : TROPICULTURA, 2011, 29, 1, 39-45, 07 pages.

28. Ndiaye A. et Sawane O., 2015. Etude des pratiques culturales locales du mil sanio (Pennisetum glaucum L.) en parcelles paysannes en Haute Casamance (Sénégal). Mémoire de Licence en Agroforesterie, département agroforesterie, université Assane Seck de Ziguinchor, 37 pages. 
29. Ndour N., Y., B., N'Diénor M., Corfini N., A., Aubry C., Masse D., Paillat J., M., Diène J., Parnaudeau V.,, lounkara S., 2016. Recyclage des déchets organiques en agriculture. Éditions Ouée, 201 6, 28 pages.

30. Ouattara L.T.S., Bationo F., Parkouda C., Dao A., Bassole I.H.N., et Diawara B., 2015. Qualité des grains et aptitude à la transformation : cas des variétés de Sorghum bicolor, Pennisetumg laucumet Zea mays en usage en Afrique de l'Ouest. In international journal of biological and chemical sciences, 9(6): 2819-2832, December 2015, 14 pages.

31. Rouw A., Rajot J.L., Schrnelzer G., 2004. Effetsde Ilapport de bouses de zébus sur les composantes du rendement du mil, sur les mauvaises herbes et sur l'encroûtement superficiel du sol au Niger.

32. Sagna P., Yade M., Sambou P. C. 2012. Migration de l'équateur météorologique, fréquences de la mousson et importance des précipitations au Sénégal en 2008 et 2009. Annales de la faculté des lettres et sciences humaines. $\mathrm{N}^{\circ}$ 42/B_2012, UCAD .

33. Segnou .j., akoa a., youmbi e., et njoya j., 2012. Effet de la fertilisation minérale et organique sur le rendement en fruits du piment (capsicum annuum 1.; solanaceae) en zone forestière de basse altitude au cameroun. 10 pages

34. Siboukeur A., 2013. Appréciation de la valeur fertilisante de différents types de fumier. Mémoire de fin d'études, département des Sciences Agronomiques, faculté des sciences de la nature et de la vie et sciences de la terre et de l'univers, université kasdi Merbah Ouargla, 78 pages.

35. Somda B. B., Ouattara B., Serme I., Pouya M. B., Lompo F., Taonda S. J. B., et Sedogo P. M., 2017. Détermination des doses optimales de fumures organo-minérales en microdose dans la zone soudanosahélienne du Burkina Faso. In : International Journal of Biological and Chimical Sciences, 11(2): 670-683, 15 pages.

36. Zeinabou H., Mahamane S., Bismarck N. H., Bado B. V., Lompo F. et André B., 2014. Effet de la combinaison des fumures organo-minérales et de la rotation niébé-mil sur la nutrition azotée et les rendements du mil au sahel. In : International Journal of Biological and Chimical Sciences, 8(4): 1620-1632, 13 pages 